\title{
Changes in Cytoplasmic Streaming during the Formation of a New Growing Tip in the Coenocytic Green Alga Bryopsis plumosa (Caulerpales, Chlorophyta)
}

\author{
Ichiro Mine*, Satoko Sekida and Kazuo Okuda \\ Graduate School of Kuroshio Science, Kochi University, \\ 2-5-1 Akebono-cho, Kochi 780-8520, Japan
}

Received August 11, 2010; accepted November 4, 2010

\begin{abstract}
Summary The coenocytic thallus of Bryopsis gametophytes is composed of a main axis and side branches, both of which have a dome-like growing tip with a clear zone of protoplasm and a subsequent cylindrical, basal cell region. Pinnate thallus is formed by regular development of side branches lateral to the main axis. It has been reported that both cytoplasmic streaming and tip growth in the alga are controlled by the microtubule cytoskeleton. We examined the physiology of the tipgrowing region in $B$. plumosa by observing changes in cytoplasmic streaming during 2 different modes of growing tip formation - the germination of spherical cells regenerated from protoplasm aggregates and the formation of side branches. In the main axes, longitudinal cytoplasmic streaming, indicating chloroplast movement, was evaluated using time-lapse videomicroscopy. In the spherical cells regenerated from protoplast aggregates, chloroplasts showed active movement before and after their germination, but showed just small wriggling motion during germination. Chloroplast motility was unchanged during the early stages of side branch formation, during which a clear zone developed in cell surface protrusion. Thus cytoplasmic streaming appeared not to be involved in the growing tip formation in $B$. plumosa and hence microtubules may play a role in the growing-tip morphogenesis in this alga via mechanisms not directly related to the cytoplasmic streaming.
\end{abstract}

Key words Chloroplast, Coenocyte, Cytoskeleton, Microtubules, Tip growth.

Tip growth is a mode of localized cell growth in plants and is found typically in a cylindrical cell with a dome-like apex. Tip-growing cells have cytoskeletons composed of actin filaments and/or microtubules, which are usually arranged longitudinally to the axes of the cells (e.g., pollen tubes, Tang et al. 1989, Lancelle and Hepler 1992, Cai et al. 1996; root hairs, Ketelaar and Emons 2000, Esseling et al. 2000; Chara rhizoids, Sievers et al. 1991). These longitudinal cytoskeletal elements are involved with cell polarization that delineates the acropetal and basipetal directions of the cell (Hishinuma et al. 1997) and also serve as the route of cytoplasmic streaming, along which cell organelles move in the longitudinal direction. Although the role of the cytoskeleton in both cytoplasmic streaming and cell growth has been well recognized in tip-growing cells through the use of cytoskeletal inhibitors (e.g., Cai et al. 1996, Esseling et al. 2000), the physiological relationship between cytoplasmic streaming and cell growth has not been examined.

A number of species of coenocytic green algae show tip growth, including gametophytes of the caulerpalean alga Bryopsis plumosa (Hudson) C. Agardh. The vegetative plant of the alga, which consists of a main axis bearing side branches, is a giant coenocyte. The main axis is elongated by tip growth. The protoplasm of the tip-growing region of the cell contains a clear zone that lacks chloroplasts (Burr and West 1970). The majority of the protoplasm is occupied by a large

\footnotetext{
* Corresponding author, e-mail: mine@kochi-u.ac.jp
} 
central vacuole, which is surrounded by a thin peripheral cytoplasm that extends throughout the remaining part of the coenocyte from the basal side of the clear zone to the cylindrical region of the cell.

Side branches form on the subapical portion of the growing tip of the main axis at intervals and then grow by their own tip growth, resulting in the regular, pinnate morphology of the thallus. Thus, the regular pattern of a growing tip formation is established during the process of side branch formation (Okuda et al. 1993). Moreover, it has been shown that protoplasts spontaneously formed by aggregation of the cell sap extruded from the coenocyte readily regenerate their cell wall to form a small spherical cell and then germinate into an upright, gametophytic thallus within a few days (Tatewaki and Nagata 1970, Pak et al. 1991). The regeneration of the protoplast or germination of the spherical cell also provides an opportunity to observe a new tip-growing region of the alga.

It has also been shown in the gametophyte of Bryopsis spp. that actin filaments and microtubules arranged longitudinally in the peripheral cytoplasm of the cylindrical part of the cell participate in cytoplasmic streaming, or the movement of chloroplasts and mitochondria, in the longitudinal direction (Menzel and Schliwa 1986 a, b). Mizukami and Wada (1983) showed that disruption of microtubules caused cessation of tip growth and irregular morphogenesis in the tipgrowing region of $B$. plumosa, indicating the involvement of the microtubule cytoskeleton in the regulation of tip growth in the cell. On the basis of the results of these studies, we propose that $B$. plumosa could be used as an experimental system to directly examine the relationship between cytoplasmic streaming and cell growth in tip-growing cells. Our hypothesis is that if cytoplasmic streaming plays an indispensable role in tip growth, there should be significant changes in the pattern of cytoplasmic streaming during the formation of a new growing tip in B. plumosa.

In the present study, we observed changes in cytoplasmic streaming, which indicate chloroplast movements, during 2 modes of formation of a new growing tip - spherical cell germination and side branch formation-in B. plumosa by using time-lapse videomicroscopy in order to determine the physiological relationship between cytoplasmic streaming and tip growth.

\section{Materials and methods}

\section{Plant materials}

Unialgal culture strain of the male gametophyte of Bryopsis plumosa was established from plants collected at Charatsunai, Muroran, Hokkaido, Japan by Dr. Tatsuya Togashi. Provasoli's enriched seawater (Provasoli 1966) was used as medium. Illumination was provided by cool fluorescent lamps at $16 \mu \mathrm{mol} \mathrm{s}^{-1} \mathrm{~m}^{-2}$ in a $14 \mathrm{~h}: 10 \mathrm{~h}$ light: dark photoregimen and the temperature was $18 \pm 2^{\circ} \mathrm{C}$. Pre-culture was maintained in a culture vessel containing $150 \mathrm{ml}$ of medium under the culture condition described above. The medium was changed every 2 to 3 weeks.

Apical segments ( 2 to $3 \mathrm{~mm}$ in length) were excised from the axes or branches with a razor blade, transferred to fresh medium, and cultured under the same culture conditions as above. The segments regenerated into normal, pinnate upright thalli composed of a main axis with many side branches around it. Small spherical cells were obtained as follows: cell sap was collected by squeezing with forceps actively growing, pre-cultured coenocytes cut with a razor blade, which was allowed to aggregate by static incubation in a depression of a thick glass slide filled with $c a .3 \mathrm{ml}$ of medium for 20 to $40 \mathrm{~min}$. Aggregated protoplasm was suspended in $c a .50 \mathrm{ml}$ of medium in a culture vessel and incubated under the culture conditions as described above.

\section{Videomicroscopy of chloroplast movement}

Plant materials, such as small spherical cells during the process of thallus regeneration and upright thalli actively producing side branches, were placed in a plastic petri dish $(60 \mathrm{~mm}$ in diameter) filled with fresh medium and observed with an Olympus CK inverted microscope through 
a LWD $20 \times$ objective lens for protoplasts or a $10 \times$ objective lens for the upright thalli. Time-lapse video images were collected. Analog video images were acquired by a CCD camera (MTV-7366, Mintron Enterprise Co. Ltd., Taipei, Taiwan) through a photographic lens (NFK $2.5 \times \mathrm{LD}$, Olympus), transformed into a digital video signal using a media converter (DVMC-DA1, Sony Corp., Tokyo, Japan) and fed into a personal computer through an IEEE 1394 interface. A timelapse digital video movie was made by the computer software MotoDV (Digital Origin Inc., Mountain View, USA) with a frame acquisition interval of $5 \mathrm{~min}$. The rate of cytoplasmic streaming was determined according to the displacement of chloroplasts on the video frames.

\section{Results}

\section{Chloroplast movement in main axes of the upright thallus}

Chloroplasts showed movement in both acropetal and basipetal directions. The cylindrical region of the main axes of the upright thallus was divided into several longitudinal zones according to the direction of chloroplast movement (Fig. 1a, b). Chloroplasts present in a longitudinal zone were moving in the same direction (acropetal or basipetal), while zones with different directions of chloroplast movement were arranged side by side. The average and standard deviation of the velocity of chloroplast movement at regions $200 \mu \mathrm{m}$ from the cell tip was $1.87 \pm 0.41 \mu \mathrm{m} \mathrm{min}^{-1}$ $(n=10)$ and $1.76 \pm 0.30 \mu \mathrm{m} \mathrm{min}^{-1}(n=10)$ in acropetal and basipetal directions, respectively, and there was no significant difference between them.

There was no chloroplast movement in the protoplasmic region near the basal side of the clear zone (arrow in Fig. 1a), forming an aggregate shown as a dark-colored protoplasm along the region. When chloroplasts that had moved in the acropetal direction arrived at this region, their movement stopped and they contributed to the chloroplast aggregate. Some chloroplasts in the aggregate moved basipetally away in the longitudinal zone of the cylindrical region of the cell. As such, the size of the chloroplast aggregate was not constant and changed according to differences between the number of chloroplasts joining and leaving the aggregate.

\section{Chloroplast movement during spherical cell germination}

Protoplasm extruded from gametophytes aggregated to form masses of protoplasm, which gradually developed a cell wall and became spherical cells with discrete outlines (Fig. 1c). The surface of the spherical cells bulged out 48 to $72 \mathrm{~h}$ after protoplasm preparation, and a clear zone was formed in the protrusion (arrows in Fig. 1d). The cells grew further by tip growth at the protrusion to form a cylindrical region of cell with a dome-like growing tip (Fig. 1e).

During the period when the protoplasm aggregates became spherical cells, active rotational movement of chloroplasts in random directions was observed throughout the aggregate or cell (data not shown). This chloroplast movement continued for approximately 36 to $72 \mathrm{~h}$ after protoplasm aggregation, and its velocity ranged from 1.2 to $3.3 \mu \mathrm{m} \mathrm{min}^{-1}$. After the rotational movement stopped, directional movement of the chloroplasts was not observed in spherical cells except for oscillatory or wriggling movement, which was detected as a random shift of the chloroplast position observed in each video frame taken at 5 min intervals (Fig. 1f). The amplitude of the shift was 2 to $3.5 \mu \mathrm{m}$. This wriggling movement continued even after the beginning of tip growth of the spherical cell at the clear zone (Fig. 1g) and was then followed by active circulation of chloroplasts throughout the cytoplasm from the tip to the base of the elongated spherical cells (Fig. 1h). The velocity of the movement was $2.3 \pm 1.0 \mu \mathrm{m} \mathrm{min}^{-1}$ ( $n=18$ in 3 germinating spherical cells).

\section{Chloroplast movement during side branch formation}

When side branches were newly formed on the lateral surface of the main axes, a small protrusion of the cell surface was formed at 200 to $500 \mu \mathrm{m}$ from the cell tip (double arrow in Fig. 


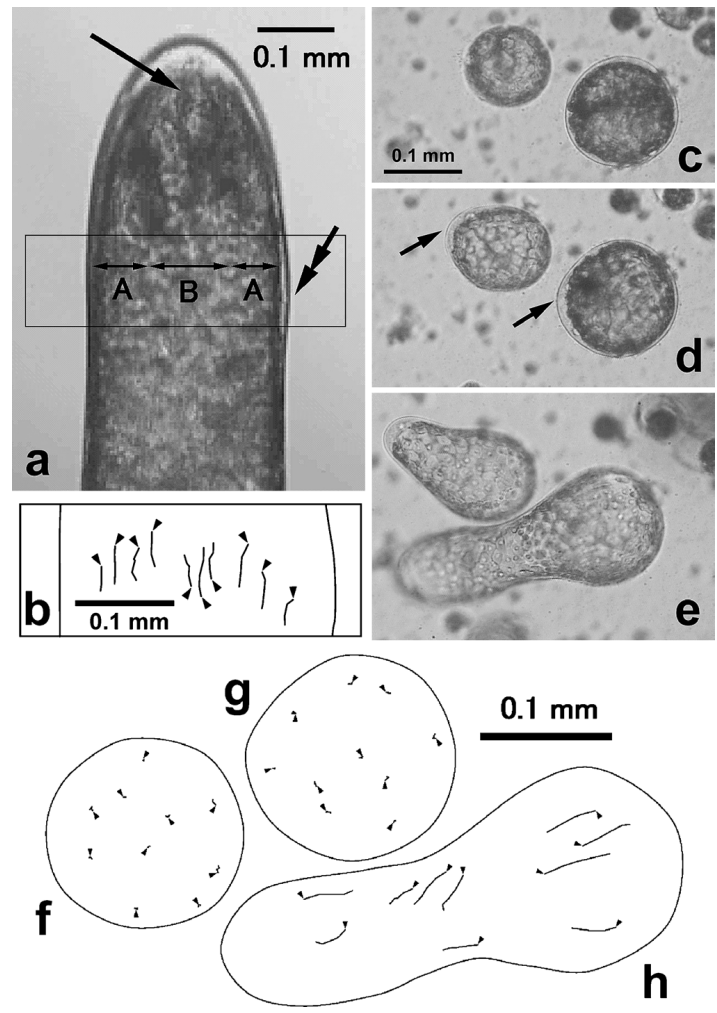

Fig. 1. The chloroplast movement in the main axis and during the spherical cell germination of Bryopsis plumosa. a, b) Growing tip of the main axis. a) Captured videomicroscopic image. The arrow indicates the apex of a growing tip and the double arrow indicates the protrusion of the lateral cell surface that is the prospective side branch. The longitudinal zone in which chloroplasts moving in the acropetal direction is indicated by " $\mathrm{A}$ " and movement in the basipetal direction is indicated by "B". A rectangular area enclosed by solid lines indicates the longitudinal position of the area shown in Fig. 1b. b) Tracks of the movement of 10 chloroplasts observed in the video image. The position of the center of each chloroplast in the video image was recorded in 5 consecutive video frames taken 10 frames after that shown in Fig 1a and connected in sequence by solid lines. The position of the chloroplast in the fifth (last) frame is indicated by an arrowhead. The rectangular area in this figure was drawn so that the position of the upper and lower sides of the area corresponds to those of the rectangular area in the Fig. 1a. The outline of the outer surface of the cell wall observed in the first frame is also traced by solid lines. Frame rate was $5 \mathrm{~min}$ per frame and, therefore, the time passed during the chloroplast movement along the whole track was $20 \mathrm{~min}$. Note that the chloroplasts in zone "A" showed upward movement, while those in "B" showed downward movement. c-h) Spherical cell germination. c-e) Captured videomicroscopic images. Fig. 1c, d, e were taken 79.5, 90 and $100.5 \mathrm{~h}$ after protoplasm preparation. c) Two spherical cells developed a cell wall and have a discrete outline. d) The spherical cells had developed a surface protrusion with a clear zone. Arrows indicate the newly developed clear zones. e) The protrusions had grown further by tip growth. In this stage, the chloroplasts began active rotational movement throughout the cell. Scale bar in Fig. 1c also applies to Fig. 1d, e. f-h) Tracks of the chloroplast movement observed in the right spherical cell in Fig. 1c. Ten chloroplasts in the cell were randomly selected in each image of Fig. $1 \mathrm{c}-$ e. Beginning from the video frame shown in Fig. $1 \mathrm{c}-\mathrm{e}$, tracks were made as in Fig. 1b. An outline of the outer surface of the cell wall observed in the first frame is also traced by solid lines. Frame rate was $5 \mathrm{~min}$ per frame. Note that the chloroplasts exhibit only oscillatory or wriggling movement in the nascent spherical cells with cell walls (Fig. 3d) and in cells with a newly developed clear zone (Fig. 3e). Active, bidirectional movement begins after the cell has grown into an elongated cell (Fig. 3f). 
1a and Fig. 2a). After the height of the protrusion reached 5 to $15 \mu \mathrm{m}$, a clear zone was formed in the protrusion (Fig. 2b, c), which was the prospective clear zone of the tip-growing side branch. The protrusion grew further by tip growth to form the cylindrical, basal part of the side branch (Fig. 2 $\mathrm{d}-\mathrm{f})$. After the length of the branch became about 40 to $60 \mu \mathrm{m}$, protoplasm containing chloroplasts and the central vacuole of the main axes moved into the cylindrical portion of the branches (Fig. $2 \mathrm{~d}$ ). The branches continued to grow into long side branches that extended obliquely from the lateral side of the main axes (Fig. 2e, f).

Longitudinal chloroplast movement in acropetal or basipetal direction along the main axes did not change around the region of side branch formation until their clear zone was produced (Fig. $2 \mathrm{~g}$, h). When the protoplasm of the main axes moved slightly into the newly formed side branches (Fig. $2 \mathrm{i}, \mathrm{j}$ ), the chloroplasts in the main axis passed through the side branches and continued to move along the longitudinal zone of the main axes. The velocity of the chloroplasts passing through the side branch was $1.70 \pm 0.30 \mu \mathrm{m} \mathrm{min}^{-1}$ ( $n=24$ in 4 side branches), which was not significantly different from the velocity in the remaining parts of the main axes.

In the elongated side branches, there were chloroplasts that entered and remained in the branches (Fig. 2k, 1). They showed uniform longitudinal movement toward the branch apex. The velocity of the movement was slower $\left(1.00 \pm 0.21 \mu \mathrm{m} \min ^{-1} ; n=9\right.$ in 2 branches) than that of chloroplasts in the main axes, and the longitudinal zones of bidirectional chloroplast movement could not be clearly observed in the newly formed side branches (Fig. $2 \mathrm{k}, 1)$.

\section{Discussion}

Cytoplasmic streaming in the cylindrical, basal region of the main axes of B. plumosa observed as chloroplast movement in the present study occurred in the longitudinal direction as reported previously (Menzel and Schliwa 1986a). Chloroplasts moved bidirectionally, i.e. in both acropetal and basipetal directions, and there were multiple longitudinal cytoplasmic zones of the main axes arranged side by side, each of which contains chloroplasts moving in the same direction (acropetal or basipetal). As mentioned above, cytoplasmic microtubules arranged longitudinally in this region have been known to be involved in the longitudinal movement of chloroplasts (Menzel and Schliwa 1986a, b, Hishinuma et al. 1997). Hishinuma et al. (1997) reported that the longitudinal microtubules of the cytoplasm in Bryopsis gametophytes maintained constant polarity - the plus end to the tip-which is inconsistent with the bidirectional chloroplast movement observed in the present study. In addition, longitudinal chloroplast movement has been shown to be inhibited by both microtubule inhibitors and actin filament inhibitors (Menzel and Schliwa 1986b). Therefore, if cortical microtubules regulate chloroplast movement in part, they may not simply serve as the route for the movement but function in cooperation with other cell motility mechanisms such as various motor proteins and actin filaments.

We also observed that the chloroplasts moving acropetally stopped their movement on the arrival in the basal side of the clear zone at cell apex and eventually restart the movement toward the basal region of the cell. Thus suspension and 180-degree turn of the chloroplast movement occurs in this apical region. As carried out in another tip-growing, giant-celled green alga Acetabularia (Sawitzky et al. 1996), observations of the arrangement of cytoskeletons in the cell apex would provide information useful for understanding the mechanism(s) controlling the abrupt changes of the organelle motility that occur in this region of $B$. plumosa gametophyte.

Contrary to our above hypothesis, cytoplasmic streaming did not seem to participate in the formation of new growing tips. The patterns of cytoplasmic streaming are different between the 2 modes of new growing tip formation: spherical cell germination and side branch formation from the main axes. Although vigorous chloroplast motility was observed before and after germination of spherical cells, no directional movement of the organelle was observed during germination. There 

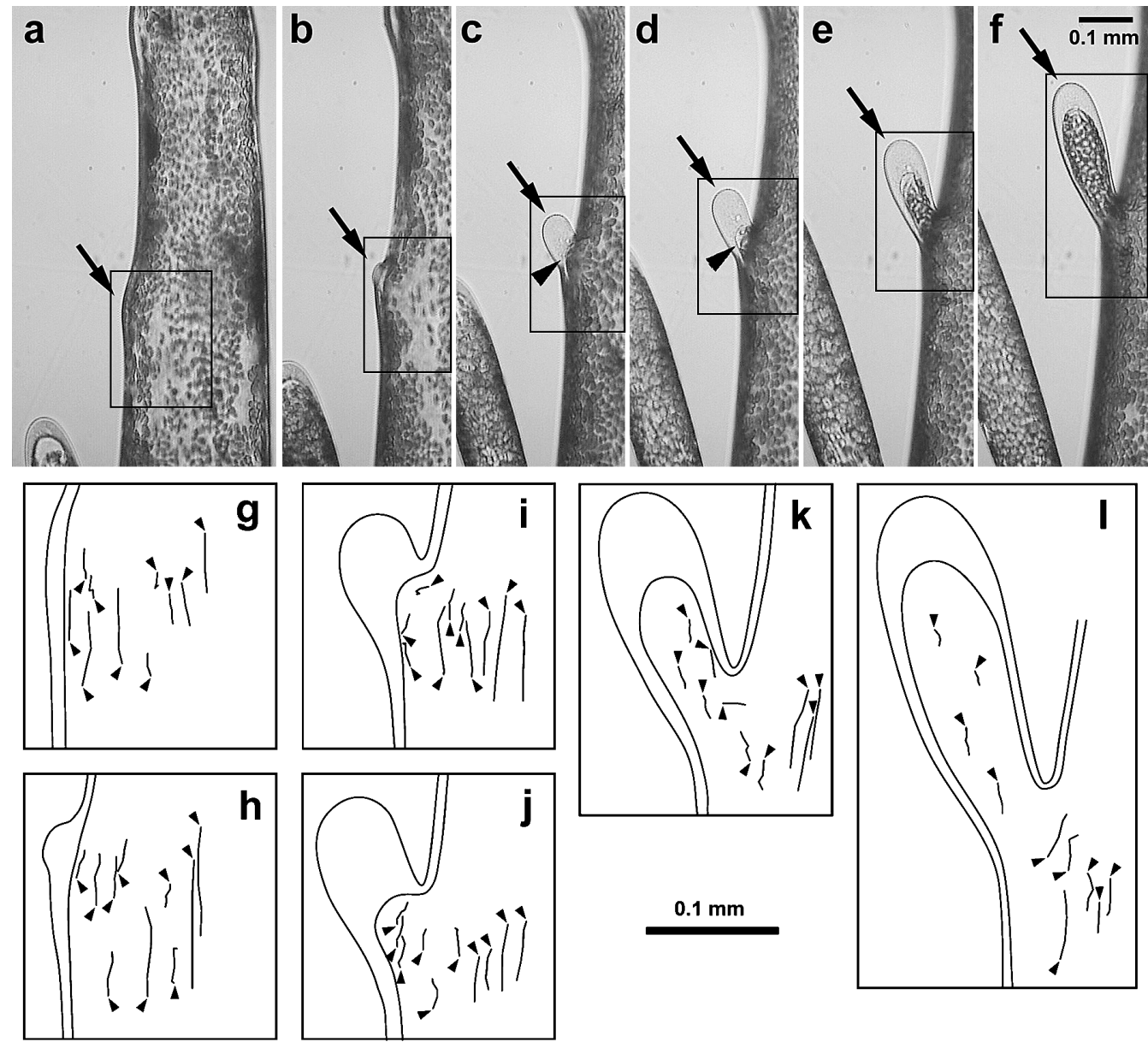

Fig. 2. Side branch formation and chloroplast movement. a-f) Captured videomicroscopic images. Fig. 2b, c, d, e and f were taken 3, 8, 15, 22 and $27 \mathrm{~h}$ after Fig. 2a, respectively. Arrows indicate the growing tip of the newly developed side branch. a) A small protrusion was formed on the lateral surface of the main axis. b) The protrusion became a discrete bulge with a developing clear zone. c) The protrusion had grown further and the protoplasm of the main axis entered slightly into the protrusion (arrowhead). d) Chloroplasts from the protoplasm of the main axis entered the protrusion with a cylindrical basal part (arrowhead). e, f) The protrusion was further extended with many chloroplasts from the main axis. Scale bar in Fig. $2 \mathrm{f}$ also applies to Fig. 2a-e. Rectangular areas enclosed by solid lines indicate the longitudinal position of the areas shown in Fig. 2g-1. g-1) Tracks of the chloroplast movement observed in the video images captured after those shown in Fig. $2 \mathrm{a}-\mathrm{f}$. Ten chloroplasts near the region of side branch formation were randomly selected in each image of the figure. Beginning from the video frame shown in Fig. 2a-f, the position of the center of each chloroplast in the video image was recorded in 5 consecutive video frames and connected in sequence by solid lines. The position of the chloroplast in the fifth (last) frame is indicated by an arrowhead. The rectangular areas in Fig. $2 \mathrm{~g}-1$ were drawn so that the positions of the upper and lower sides of the areas correspond to those of the rectangular areas in Fig. 2a-f. Outlines of the cell wall and clear zone observed in the first frame are also traced by solid lines. Frame rate was 5 min per frame. Note that the bidirectional chloroplast movement in the main axis continued during side branch formation (Fig. $2 \mathrm{~g}-1$ ), and the slower movement of the chloroplasts that had entered in the side branch toward the branch tip (Fig. 2k, 1). 
was no change in chloroplast movement in the main axes during the early stages of side branch formation. Thus, there is no apparent relationship between cytoplasmic streaming occurring in the protoplasm beneath the clear zone and the formation of new growing tips in B. plumosa. It has been shown that treatment with cytoskeleton inhibitors, such as inhibitors of actin filaments and microtubules, also suppresses tip growth in the cells of many plants including Bryopsis (Mizukami and Wada 1983). Therefore, cell activity dependent on such cytoskeletal elements might participate in the regulation of tip growth in these cells. In Bryopsis, according to the observations in the present study, microtubules would control the tip growth through a mechanism that is not directly related to cytoplasmic streaming.

The observation of fine structures of the Bryopsis gametophyte showed that the clear zone of the main axes is divided into an apical zone containing vesicles and endoplasmic reticulum and a subapical zone containing other colorless organelles such as Golgi bodies and mitochondria (Burr and West 1970). Cytoskeletal elements may play a role in the orderly construction of the clear zone and/or vesicle transportation between organelles and plasma membranes, which participate in the formation of a new cell surface during tip growth of the cell. For example, since the density of vesicles in the tip-growing region of pollen tubes is increased with cytochalasin B treatment, the actin cytoskeleton may play a role in vesicle transportation and/or exocytosis in the very tip of the cell (Picton and Steers 1983). To test such a possibility in Bryopsis, it is necessary to analyze the movement of fine structures within the clear zone and outermost layer of the peripheral cytoplasm during tip growth and the formation of newly growing tips in living cells.

\section{References}

Burr, F. A. and West, J. A. 1970. Light and electrion microscopic observations on the vegetative and reproductive structures of Bryopsis hypnoides. Phycologia 9: 17-37.

Cai, G., Moscatelli, A., Del Casino, C. and Cresti, M. 1996. Cytoplasmic motors and pollen tube growth. Sex. Plant Reprod. 9: 59-64.

Esseling, J., de Ruijter, N. and Emons, A. M. C. 2000. The root hair actin cytoskeleton as backbone, highway, morphogenetic instrument and target for signalling. In: Ridge, R.W. and Emons, A. M. C. (eds.). Root Hairs; Cell and Molecular Biology. Springer-Verlag, Tokyo. pp. 29-52.

Ketelaar, T. and Emons A. M. C. 2000. The role of microtubules in root hair growth and celluose microfibril deposition. In: Ridge, R.W. and Emons, A. M. C. (eds.). Root Hairs; Cell and Molecular Biology. Springer-Verlag, Tokyo. pp. 17-28.

Hishinuma, T., Tsubura, H. and Wada, S. 1997. Cell polarity in a giant unicellular green alga, Bryopsis plumosa. In: Bonotto, S. and Berger, S. (eds.) ATTI Proceeding, Symposium Ecology and Biology of Giant Unicellular Algae. Museo Regionale di Scienze Naturali, Torino. pp. 103-118.

Lancelle, S. A. and Hepler, P. K. 1992. Ultrastructure of freeze-substituted pollen tubes of Lilium longiflorum. Protoplasma 167: 215-230.

Menzel, D. and Schliwa, M. 1986a. Motility in the siphonous green alga Bryopsis I. Spatial organization of the cytoskeleton and organelle movements. Eur. J. Cell Biol. 40: 275-285.

— and - 1986b. Motiliy in the siphonous green alga Bryopsis II. Chloroplast movement requires organized arrays of both microtubules and actin filaments. Eur. J. Cell Biol. 40: 286-295.

Mizukami, M. and Wada, S. 1983. Morphological anomalies induced by antimicrotubule agents in Bryospsis plumosa. Protoplasma 114: 151-162.

Okuda, K., Matsusaki, Y. and Mizuta, S. 1993. Cytomorphogenesis in coenocytic green algae. II. Dynamics of nuclei during vegetative and gametogenetic differentiation in Bryopsis corticulans. Mem. Fac. Sci., Kochi Univ., Ser. D. Biol. 14: 57-67.

Pak, J. Y., Solorzano, C. Arai, M. and Nitta, T. 1991. Two distinct steps for spontaneous generation of subprotoplasts from a disintegrated Bryopsis cell. Plant Physiol. 96: 819-825.

Picton, J. M. and Steer, M. W. 1983. Membrane recycling and the control of secretory activity in pollen tubes. J. Cell Sci. 63: 303-310.

Provasoli, L. 1966. Media and prospects for the cultivation of marine algae. In: Watanabe, A. and Hattori, A. (eds.). Cultures and Collections of Algae. Japanese Society of Plant Physiologists, Tokyo. pp. 63-75.

Sawitzky, H., Willingale-Theune, J. and Menzel, D. 1996. Improved visualization of F-actin in the green alga Acetabularia 
by microwave-accelerated fixation and simultaneous FITC-Phalloidin staining. Histochem. J. 28: 353-60.

Sievers, A., Buchen, B., Volkmann, D. and Hejnowicz, Z. 1991. Role of the cytoskeleton in gravity perception. In: Lloyd,

C.W. (ed). The Cytoskeletal Basis of Plant Growth and Development. Academic Press, London. pp. 169-182.

Tang, X. J., Lancelle, S. A. and Hepler, P. K. 1989. Fluorescence microscopic localization of actin in pollen tubes: comparison of actin antibody and phalloidin staining. Cell Motil. Cytoskeleton 12: 216-24.

Tatewaki, T. and Nagata, K. 1970. Surviving protoplasts in vitro and their development in Bryopsis. J. Phycol. 6: 401-403. 